\title{
ON NONHOMOGENEOUS p-LAPLACIAN ELLIPTIC EQUATIONS INVOLVING A CRITICAL SOBOLEV EXPONENT AND MULTIPLE HARDY-TYPE TERMS
}

\author{
SOFIANE MESSIRDI and ATIKA MATALLAH
}

\begin{abstract}
In this paper, we consider a class of nonhomogeneous $p$-Laplacian elliptic equations with a critical Sobolev exponent and multiple Hardy type terms. By the Ekeland variational principale on a Nehari manifold and the mountain pass lemma, we prove the existence of multiple solutions, under sufficient conditions on the data and the considered parameters.
\end{abstract}

MSC 2010. 35J20, 35J50, 35B33.

Key words. Concentration compactness principle, critical Sobolev exponent, Ekeland's variational principle, Hardy inequality, Multi-singular potentials, Palais-Smale condition.

\section{REFERENCES}

[1] B. Abdellaoui, V. Felli and I. Peral, Existence and nonexistence for quasilinear equations involving the p-Laplacian, Boll. Unione Mat. Ital., 9-B (2006), 445-484.

[2] M. Bouchekif and S. Messirdi, On nonhomogeneous elliptic equations with critical Sobolev exponent and prescribed singularities, Taiwanese J. Math., 20 (2016), 431-447.

[3] J. Chen, Multiple positive solutions for a semilinear equation with prescribed singularity, J. Math. Anal. Appl., 305 (2005), 140-157.

[4] I. Ekeland, On the variational principle, J. Math. Anal. Appl., 17 (1974), 324-353.

[5] P. Han, Quasilinear elliptic problems with critical exponents and Hardy terms, Nonlinear Anal., 61 (2005), 735-758.

[6] G.H. Hardy, J.E. Littlewood and G. Polya, Inequalities, Cambridge University Press, 1988.

[7] T.-S. Hsu, Multiple positive solutions for quasilinear elliptic problems involving concaveconvex nonlinearities and multiple Hardy-type terms, Acta Math. Sci., 33 (2013), 13141328 .

[8] E. Jannelli, The role played by space dimension in elliptic critical problems, J. Differential Equations, 156 (1999), 407-426.

[9] D. Kang, On the singular critical quasilinear problems in $\mathbb{R}^{\mathbb{N}}$, Nonlinear Anal., 69 (2008), $3577-3590$.

[10] H. Liu, Multiple positive solutions for a quasilinear elliptic equation involving singular potential and critical Sobolev exponent, Nonlinear Anal., 71 (2009), 1684-1690.

[11] P.L. Lions, The concentration-compactness principle in the calculus of variations, the limit case (I), Rev. Mat. Iberoam., 1 (1985), 145-201.

The authors thank the referee for his helpful comments and suggestions.

DOI: 10.24193/mathcluj.2019.1.05 
[12] P.L. Lions, The concentration-compactness principle in the calculus of variations, the limit case (II), Rev. Mat. Iberoam., 1 (1985), 45-121.

[13] G. Tarantello, On nonhomogeneous elliptic equations involving critical Sobolev exponent, Ann. Inst. H. Poincaré Anal. Non Linéaire, 9 (1992), 281-309.

Received April 2, 2018

Accepted October 16, 2018
University of Mostaganem

Faculty of Exact Sciences

Department of Mathematics and Informatics

Laboratory of Fundamental and Applicable Mathematics of Oran (LMFAO)

Mostaganem, Algeria

E-mail: messirdi.sofiane@hotmail.fr

Ecole Supérieure de Management de Tlemcen Algeria

E-mail: atika_matallah@yahoo.fr 